\title{
Out on the full
}

Cancers that arise as a result of mutation in either BRCA1 or BRCA2 are deficient in specific aspects of DNA repair and are sensitive to drugs that exploit this weakness, unless resistance occurs. Two groups have now identified one mechanism of resistance: the re-expression of a functional form of BRCA2; in some cases almost the full-length protein.

BRCA2, along with the recombinase RAD51, is involved in the repair of DNA double-strand breaks through homologous recombination (HR). Loss of this pathway makes BRCA2-mutant tumours sensitive to DNA cross-linking agents, such as the platinum-based drugs, and also to inhibition of poly (ADP-ribose) polymerase 1 (PARP1), an enzyme involved in DNA repair. To examine how resistance to these drugs develops, Alan Ashworth and colleagues, and Toshiyasu Taniguchi and colleagues, examined drug-resistant clones produced from the BRCA2deficient pancreatic cancer cell line CAPAN1. This cell line expresses a mutant BRCA2 with a frameshift mutation (6174delT) that results in a truncated form of the protein that lacks the carboxyl terminus and cannot bind RAD51. Fluorescence in situ hybridisation carried out by both groups indicated that CAPAN1 cells have at least two copies of the mutant 6174delT BRCA2 gene.

Ashworth and colleagues produced PARP-inhibitor-resistant (PIR) CAPAN1 clones through exposure to the PARP inhibitor KU0058948 and found most of the clones were able to form RAD51 foci after treatment with the PARP inhibitor or exposure to X-rays. Although these clones still expressed the 6174delT mutant protein, they also expressed new BRCA2 proteins that had both the amino and carboxyl termini. Further examination indicated that some had increased copies of the BRCA2 gene and intragenic deletions of varying size that deleted the frameshift mutation and restored expression of the C-terminal portion of the protein. Ashworth and colleagues found that the PIR clones were also resistant to treatment with platinum drugs.

Taniguchi and colleagues examined 14 cisplatin-resistant CAPAN1 clones and found that 7 re-expressed BRCA2 at lengths close to that of the wild type. They found that intragenic mutations had arisen in duplicated copies of BRCA2 that corrected the 6174 delT frameshift mutation. These mutant clones formed RAD51 foci when the cells were treated with ionizing radiation, and were also resistant to PARP inhibitors. Interestingly, the remaining 7 resistant clones showed no change in BRAC2 expression and remained sensitive to PARP inhibitors, indicating an alternative mechanism(s) of resistance to cisplatin.

Both groups investigated tumours from a small number of patients with ovarian cancer with known mutations in BRCA2 and found that resistance to platinum, part of the standard treatment for ovarian cancer, can be associated with the re-expression of a functional form of BRCA2.
So, it seems that it will be important to determine the mechanisms of resistance in patients with $B R C A 2$ mutant tumours, as those that do not show changes in BRCA2 and are resistant to platinum drugs might remain sensitive to PARP inhibitors once these agents reach the clinic. Ironically, as both papers point out, it might be the case that the DNA repair defects caused by mutation of BRCA2 increase the likelihood of mutations that lead to the deletion of the 6174delT frameshift and drug resistance.

Nicola McCarthy

ORIGINAL RESEARCH PAPERS Edwards, S. L et al. Resistance to therapy caused by intragenic deletion in BRCA2. Nature 451, 1111-1115 (2008) | Sakai, W. et al. Secondary mutations as a mechanism of cisplatin resistance in BRCA2 mutated cancers. Nature 451, 1116-1120 (2008)

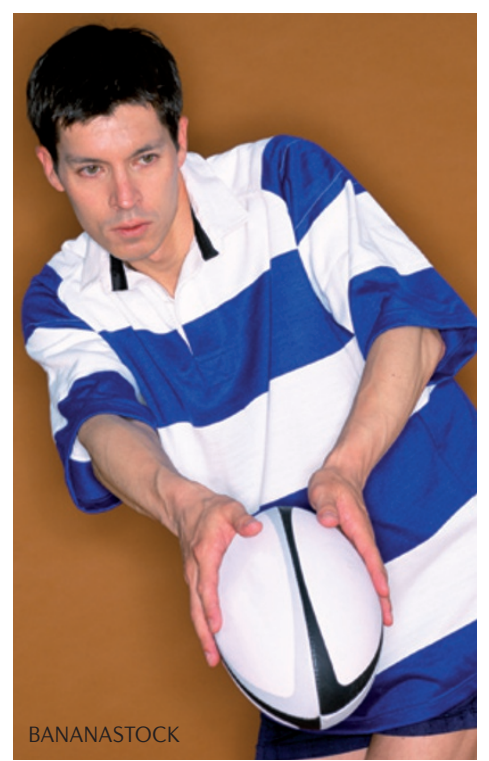

